\title{
Estudio de parámetros hídricos foliares en trigo (Triticum aestivum L.) y su uso en selección de genotipos resistentes a sequía
}

\author{
Leaf water parameters of wheat (Triticum aestivum L.) and their use in the selection \\ of drought resistant genotypes
}

MAURICIO ORTIZ, HERMAN SILVA, PAOLA SILVA \& EDMUNDO ACEVEDO ${ }^{1}$

Laboratorio de Relación Suelo-Agua-Planta, Facultad de Ciencias Agronómicas, Universidad de Chile, Casilla 1004, Santiago, Chile; e-mail'1: eacevedo@abello.dic.uchile.cl

\begin{abstract}
RESUMEN
Los parámetros hídricos foliares $\psi$ s (potencial de solutos), CRA (contenido relativo de agua) y AO (ajuste osmótico) permiten caracterizar la respuesta de las plantas frente al estrés hídrico e identificar aquellos genotipos mejor adaptados. Sin embargo, estos parámetros presentan una gran influencia del medioambiente lo que dificulta su análisis. En este trabajo se planteó la hipótesis de que es posible caracterizar y seleccionar genotipos resistentes a sequía en base a la evolución de los parámetros hídricos foliares en campo. Se cultivaron 31 genotipos de trigo (Triticum aestivum L.) en dos ensayos de campo, uno regado y otro no regado que recibió sólo 218,3 $\mathrm{mm}$ de lluvia invernal. El diseño fue de bloques al azar con dos repeticiones. Entre los 77 y 121 días después de la emergencia (DC 41 a DC 77) se realizaron cinco muestreos de CRA y $\psi$ s en hojas bandera hidratadas ( $\psi$ sh) en cada repetición de cada ensayo (con un total de 10 observaciones por ensayo). Las repeticiones se muestrearon en días alternos, con un intervalo de $24 \mathrm{~h}$ entre las 12:00 y las 14:00 h. A la cosecha se midió biomasa, rendimiento y componentes de rendimiento. Las observaciones de CRA y $\psi$ sh fueron altamente afectadas por factores ambientales (ca. $80 \%$ de la suma de cuadrados) y no estuvieron correlacionadas entre repeticiones de un mismo ensayo. Con fines de análisis se consideró cada observación como independiente ( 10 observaciones de cada parámetro hídrico foliar por genotipo) y se realizaron regresiones lineales del valor de cada parámetro hídrico foliar de cada genotipo sobre el promedio de todos los genotipos en el momento de muestreo. Cada genotipo se caracterizó por el valor medio del parámetro hídrico foliar y la pendiente de la regresión. Además se estimó el ajuste osmótico (AO) de cada genotipo utilizando tres métodos propuestos en la literatura. Se hicieron dos selecciones de genotipos, una consideró la pendiente y el promedio de $\psi$ sh, CRA y AO, y otra que consideró la pendiente y el promedio de $\psi$ sh y CRA. Luego se correlacionó el orden de los genotipos de ambas selecciones con los ordenes establecidos para los métodos de calculo de $\mathrm{AO}$ y se estableció que el orden que considera la pendiente y el promedio de $\psi$ sh, CRA y AO se correlaciona con los ordenes establecidos por los tres métodos de cálculo de AO. Los parámetros hídricos foliares CRA, $\psi$ sh y AO no estuvieron correlacionados con el rendimiento bajo estrés hídrico. La pendiente de $\psi$ sh se correlacionó negativamente con el rendimiento, lo que indica que el AO permite a la planta sobrevivir al estrés pero no tener mayor rendimiento. Se concluye que con la metodología utilizada es posible seleccionar genotipos resistentes a sequía en base a la pendiente y el promedio de los parámetros $\psi$ sh, CRA y AO obtenidos en campo, removiendo parte del ruido ambiental.
\end{abstract}

Palabras clave: trigo, parámetros hídricos foliares, rendimiento.

\begin{abstract}
The leaf water parameters $\psi s$ (solute potential), RWC (relative water content) and OA (osmotic adjustment) characterize the response of plants to water stress and presumably allow the identification of better adapted genotypes. These parameters, however, are highly influenced by the environment what makes their analysis difficult. In this work we hypothesize that it is possible to characterize and select drought resistant wheat genotypes based on the field value of the leaf water parameters using the appropriate analytical techniques. Thirty one wheat (Triticum aestivum L.) genotypes were grown in two field trials, one irrigated and one non-irrigated that received $218.3 \mathrm{~mm}$ of winter rain. The statistical design was a randomized complete block with two replicates. Between 77 and 121 days after emergence (DC 41 to DC 77), five samplings of relative water content (RWC) and solute potential of hydrated flag leaves ( $\psi \mathrm{sh}$ ) were made in each replication of each trial (10 observations per trial). The replicates were sampled in alternate days with a 24-h interval between 12:00 and 14:00 h. During harvest, biomass, yield and yield components were measured. The observations both of RWC and $\psi$ sh were highly affected by environmental factors (ca. $80 \%$ of the sum of squares) while they were not correlated between replications within each trial. For analysis purpose, each observation was considered as independent ( 10 observations for each leaf water parameter per genotype) and linear regressions were made of each leaf water parameter value of each genotype on the mean of all the genotypes at the time of sampling. Each genotype was characterized by its average value of leaf water parameter and the regression slope. The osmotic adjustment (OA) of each genotype was estimated by utilizing different methodologies proposed in the literature. Two genotype selections were made, one using the slope and average of $\psi$ sh, RWC and OA, and one that considered the
\end{abstract}


slope and average of $\psi \mathrm{sh}$ and RWC. The order of the genotypes of both selection criteria were then correlated with orders established by the three methods of calculation of $\mathrm{OA}$, this established that the order that considers the slope and the average of $\psi \mathrm{sh}, \mathrm{RWC}$ and OA, correlated with the order established by the three methodologies to calculate OA. This points out that it is possible to use the slope criteria in the selection of genotypes that adjust osmotically. Leaf water parameters RWC, $\psi$ sh and OA were not correlated to yield. The slope of $\psi$ sh was negatively related to yield pointing to a survival role of OA. It is concluded that it is possible to select drought resistant genotypes using the mean and slope of $\psi \mathrm{sh}, \mathrm{RWC}$ and OA by removing part of the environmental noise.

Key words: wheat, leaf water parameters, yield.

\section{INTRODUCCIÓN}

La resistencia a la sequía en las especies de cultivo está asociada al grado en que éstas regulan el contenido de agua y el potencial hídrico foliar durante el estrés. La capacidad de una planta para mantener su estado hídrico a medida que el potencial hídrico del suelo disminuye constituye una adaptación al déficit hídrico (Acevedo et al. 1998). Esta capacidad se refleja en el contenido relativo de agua (CRA), en el potencial de solutos ( $\psi s)$ de los tejidos y en el ajuste osmótico (AO).

El CRA (Barrs \& Weatherley 1962) es la expresión más usada para medir el nivel de agua de un tejido. Es una medida del contenido de agua del tejido respecto del total de agua que éste puede almacenar, se expresa como porcentaje y permite conocer el estado hídrico de la planta. Se relaciona con el potencial hídrico porque éste y sus componentes, potencial de presión y de solutos, son función del volumen de agua del protoplasma.

El potencial de solutos o potencial osmótico es una expresión que refleja la actividad de los solutos en solución. Para comparar $\psi$ s de diferentes genotipos se debe estandarizar el nivel hídrico del tejido al que se expresa, lo que generalmente se hace a máximo turgor (CRA $\cong 100 \%$ : $\psi \mathrm{sh}$, $\psi$ s 100) o a turgor cero (CRA $\left.\cong 70 \%: \psi \mathrm{s}_{0}\right)$. Para estimar $\psi$ sh se pueden hidratar los tejidos flotándolos en agua destilada previo a la determinación del potencial de solutos o bien regar las plantas la noche anterior al muestreo (Blum 1989); otra manera de estimar el potencial de solutos a turgor máximo es corrigiendo el valor de $\psi$ s de hojas sin hidratar, por el valor de CRA del tejido y obtener $\psi$ s 100 (Wilson et al.1979).

El ajuste osmótico es un proceso que consiste en la acumulación activa de solutos en tejidos de la planta en respuesta a una disminución de la disponibilidad de agua. Con ello disminuye el potencial de solutos y el potencial hídrico total de tallos, hojas, y raíces (Acevedo 1975, Turner \& Jones 1980, Girma \& Krieg 1992). Como resultado, las plantas pueden absorber agua a potenciales hídricos del suelo menores y mantener la presión de turgor y la actividad fisiológica rela- cionada al contenido hídrico en los tejidos (Acevedo 1975, Hsiao et al. 1976, Blum \& Sullivan 1986, Ludlow et al. 1990, Acevedo et al. 1999). El trigo es una especie que ajusta osmóticamente y tiene variación genética para este carácter (Morgan 1983, Blum et al. 1999). Hay trabajos que señalan que el ajuste osmótico está relacionado positivamente al rendimiento bajo condiciones de estrés hídrico en este cereal, por lo que mejora el rendimiento en grano bajo estrés (Morgan 1995, El Hafid et al. 1998, Blum et al. 1999). Además de extraer más agua del suelo, los genotipos de trigo con alto ajuste osmótico pueden producir alta biomasa de raíces, alta densidad y longitud de raíces, y tener mayor transpiración (Morgan 1984).

Existen diferentes métodos para estimar el ajuste osmótico, todos requieren que material vegetal en un determinado estado de desarrollo se someta a un ciclo de estrés hídrico en forma lenta. El ajuste osmótico se puede estimar a partir de la diferencia del potencial de solutos expresado a turgor máximo en hojas de plantas estresadas menos el potencial de solutos de hojas de plantas no estresadas, o bien a través de la evolución del potencial de solutos a lo largo de un periodo de déficit en que se corrija por la disminución de $\psi s$ producto de la deshidratación de los tejidos (disminución de CRA), que no constituye ajuste osmótico (Morgan 1992).

La correlación positiva observada entre ajuste osmótico y tolerancia a sequía ha sugerido la posibilidad de usar esta característica como un criterio de selección para rendimiento en condiciones de sequía (Rekika et al. 1998). Pero el ajuste osmótico, al igual que otros parámetros hídricos, presenta grandes fluctuaciones con las condiciones ambientales, como temperatura, intensidad de luz, concentración de $\mathrm{CO}_{2}$ y otras, dificultando la caracterización de los genotipos en relación a estos parámetros.

La variabilidad en la respuesta de los parámetros hídricos foliares plantea problemas en la interpretación del comportamiento de éstos frente al estrés hídrico y frente al rendimiento, lo que se solucionaría al remover parte del efecto ambiental. Este trabajo plantea la hipótesis de que es posible caracterizar genotipos resistentes a se- 
quía en base a la evolución de los parámetros hídricos foliares en campo si se usan las técnicas apropiadas.

Los objetivos de este trabajo fueron los siguientes: (i) Estudiar la variación de parámetros hídricos foliares frente al estrés hídrico bajo condiciones de campo; (ii) Encontrar la forma de análisis que lleve a resultados confiables, minimizando los efectos ambientales; (iii) Probar si el análisis permite la identificación de genotipos superiores en términos de relaciones hídricas; (iv) analizar la relación entre los parámetros hídricos foliares y rendimiento bajo estrés hídrico.

\section{MATERIALES Y MÉTODOS}

\section{Sitio de estudio}

El trabajo se realizó en el Campo Experimental Antumapu de la Facultad de Ciencias Agronómicas de la Universidad de Chile ( $33^{\circ} 40^{\prime} \mathrm{S}, 70^{\circ} 38^{\prime} \mathrm{O}$; $420 \mathrm{~m}$ de altitud).

El suelo de Antumapu es de origen aluvial, pertenece cartográficamente a la serie de suelos Santiago (Chile, Comisión Nacional de Riego 1981), que es miembro de la familia "coarse loamy over sandy, skeletal, mixed, thermic typic xerochrepts". Este suelo se caracteriza por tener una profundidad media de $60 \mathrm{~cm}$, se presenta en una topografía plana, su textura es franco arenosa y tiene buen drenaje. La capacidad de campo (CC) es de $225 \mathrm{~mm}$ y el punto de marchitez permanente (PMP) es de $117 \mathrm{~mm}$ en los $60 \mathrm{~cm}$ de suelo. El contenido de agua del suelo se obtuvo mediante determinaciones gravimétricas a $30 \mathrm{~cm}$ de profundidad, que se realizaron cada 15 días.

El clima es de tipo templado mesotermal estenotérmico, mediterráneo, semiárido. El régimen térmico se caracteriza por temperaturas medias que varían entre una máxima de enero de $28,7{ }^{\circ} \mathrm{C}$ y una mínima de julio de $3,4{ }^{\circ} \mathrm{C}$. El régimen hídrico tiene precipitación invernal con una media anual de $330 \mathrm{~mm}$, un déficit hídrico de $1.030 \mathrm{~mm}$ y un período seco de 8 meses (septiembre a abril). El clima presenta veranos calurosos y secos e inviernos fríos, correspondiendo al clima tipo valle central de Chile (Santibañez \& Uribe 1990).

\section{Diseño experimental y condiciones de cultivo}

Se utilizaron 31 genotipos de trigo (Triticum aestivum L.) primaverales de diferentes procedencias (Tabla 1). Entre estos hay genotipos de alto $\mathrm{AO}$, de bajo AO más 10 genotipos naciona- les. El experimento tuvo dos ensayos independientes, uno regado y el otro de secano. El ensayo regado se regó por aspersión cada vez que la humedad a $30 \mathrm{~cm}$ de profundidad del suelo llegó a $50 \%$ de la humedad aprovechable. El ensayo de secano no se regó en toda la temporada, lo que generó déficit hídrico a pesar de las lluvias invernales $(218,3 \mathrm{~mm}$ durante el desarrollo del ensayo).

El diseño experimental de cada ensayo fue de bloques completos al azar con dos repeticiones. La unidad experimental fue una parcela de $4 \mathrm{~m}$ de largo por 1,6 $\mathrm{m}$ de ancho (ocho hileras separadas por $0,2 \mathrm{~m}$ ). La siembra se realizó entre el 8 y 9 de julio de 1999. La dosis de semilla fue de $12 \mathrm{~g} \mathrm{~m}^{-2}$. La fertilización consistió en la aplicación de 100 $\mathrm{kg} \mathrm{ha}^{-1}$ de nitrógeno (urea) y $80 \mathrm{~kg} \mathrm{ha}^{-1}$ de $\mathrm{P}_{2} \mathrm{O}_{5}$ (Super fosfato triple) a la siembra, y $100 \mathrm{~kg} \mathrm{ha}^{-1}$ de nitrógeno (urea) aplicados a fines de macolla (14 de septiembre). Las malezas se controlaron mediante la aplicación de Ajax 50 PE (Metsulfuronmetil), los insectos, fundamentalmente pulgones, se controlaron con la aplicación de Lorsban (Clorpirifos) y las enfermedades se previnieron usando Tilt (Propiconazol). Todos los agroquímicos se usaron en las dosis recomendadas por el fabricante.

Parámetros hídricos foliares: determinación del potencial de solutos ( $\psi s$ ) y contenido relativo de agua (CRA)

En las plantas se realizaron mediciones de potencial de solutos ( $\psi$ s), potencial de solutos en hojas hidratadas ( $\psi$ sh) y contenido relativo de agua (CRA) en hojas banderas de cada uno de los genotipos de trigo entre los días 77 y 121 después de la emergencia del cultivo. Las observaciones se realizaron entre las 12:00 y 14:00 h, tanto en el ensayo regado como en el no regado. El período de muestreo correspondió al período de crecimiento de la espiga (DC 41 a DC 77, Zadoks et al. 1974), coincidente con el tiempo al que comenzó a disminuir la humedad aprovechable en el ensayo no regado.

Se colectaron hojas banderas, al azar, de cuatro plantas pertenecientes a las hileras centrales de cada parcela, se realizaron 10 muestreos (cinco en cada repetición). Debido a la cantidad de muestras a procesar los muestreos de las hojas bandera de los genotipos se realizaron en las repeticiones de los ensayos en días alternos. De las hojas muestreadas sólo se utilizó el tercio central, al que se le eliminó el nervio medio, quedando dos grupos de mitades de hojas. A una parte del material se le determinó el peso fresco (PF) y luego se 
trasladó a placas petri con agua destilada por $3 \mathrm{~h}$, tiempo determinado previamente para que el tejido alcance el peso túrgido (PT), finalmente el peso seco (PS) se obtuvo sometiendo las muestras a estufa de aire forzado a $70{ }^{\circ} \mathrm{C}$ durante $48 \mathrm{~h}$. Con estos datos se calculó CRA de acuerdo a (Barrs \& Weatherley 1962):

$$
\mathrm{CRA}=(\mathrm{PF}-\mathrm{PS}) /(\mathrm{PT}-\mathrm{PS})
$$

El potencial de solutos se determinó en muestras de hojas sin hidratar ( $\psi s)$ e hidratadas ( $\psi s h$ ). Las primeras fueron congeladas en nitrógeno líquido, y luego descongeladas para extraer una muestra de savia por presión en la que se determinó la osmolalidad mediante un osmómetro Vapro 5520 (Wescor). El valor obtenido se transformó a potencial de solutos mediante la formula:

$$
\psi \mathrm{s}=-\mathrm{n} * \mathrm{~T} * \mathrm{~K}
$$

donde $\mathrm{n}$ es la osmolalidad, $\mathrm{T}$ es la temperatura absoluta y $\mathrm{K}$ es la contante de los gases. Las muestras de tejido hidratadas se secaron con papel absorbente y, luego de obtener el PT, se les determinó el valor de ysh de la forma descrita anteriormente. En el material sobrante se determino el PS.

El valor de $\psi$ s se corrigió por el CRA determinado en la otra fracción del material, de manera de llevar $\psi s$ a turgor máximo, obteniéndose el ४s100 mediante la relación (Wilson et al. 1979):

$$
\psi \mathrm{s} 100=\psi \mathrm{s}[(\mathrm{CRA}-\mathrm{B}) /(100-\mathrm{B})]
$$

en que B corresponde al agua apoplástica del tejido foliar que se supuso de $15 \%$.

Origen y nombre/pedigree de los genotipos de trigo usados en este trabajo. La numeración corresponde a aquella mantenida en el Laboratorio de Relación Suelo-Agua-Planta de la Universidad de Chile

\begin{tabular}{|c|c|c|}
\hline Genotipo & Origen & Nombre/Pedigree \\
\hline 3 & CIMMYT & PFAU/SERI//BCM85295-0101Y-2M-OY-OM-1Y-OM \\
\hline 23 & CIMMYT & KAUZ CM67458-4Y-1M-3Y-1M-2Y-OB \\
\hline 36 & CIMMYT & PRL/PVN TE82.0009-18Y-025H-OSY-BM-4M-OY \\
\hline 45 & ICARDA & GV/Ald'S' L 882-1AP-OAP-2AP-OAP \\
\hline 48 & ICARDA & P106.19//Soty/Jt*3 L 489-2L-1AP-2AP-1AP-1AP-OAP \\
\hline 63 & CIMMYT & MÉXICO 82 \\
\hline 70 & CIMMYT & GV/ALD'S', L.882-1AP-OAP-2AP-OAP \\
\hline 71 & CIMMYT & TEMPORALERA \\
\hline 74 & CIMMYT & PWYT-A4= ? ?NR86-I = BAU', S', CM59123-3M-1Y-2M-1Y-2M-2Y-OM \\
\hline 76 & CIMMYT & Vulture's' \\
\hline 92 & CIMMYT & Katya A-1 \\
\hline 93 & CIMMYT & PRL/VEE\#6 \\
\hline 101 & CIMMYT & Var. 130 (R . RODRIGUEZ) \\
\hline 105 & CIMMYT & ATILA \\
\hline 118 & CIMMYT & ULC/PVN//TAN/3/BUN \\
\hline 125 & CIMMYT & SHA4/URES//KAUZ \\
\hline 127 & CIMMYT & OPATA*2/WULP(CM100684-J-OB-OY) \\
\hline 133 & CIMMYT & OASIS \\
\hline 138 & CIMMYT & $\mathrm{CNO} 79 * 2 / \mathrm{PRL}$ \\
\hline 142 & CIMMYT & $\mathrm{BCN}$ \\
\hline 145 & INIA & Coyau INIA \\
\hline 146 & INIA & Canelo INIA \\
\hline 147 & INIA & Nobo INIA \\
\hline 148 & SNA & SNA 208 \\
\hline 149 & INIA & Sofia U. UC \\
\hline 150 & INIA & Maqui INIA \\
\hline 151 & INIA & Queñoa INIA \\
\hline 152 & INIA & Huayun INIA \\
\hline 153 & INIA & Millaleu INIA \\
\hline 154 & INIA & Huanil INIA \\
\hline 155 & Australia & Q19 \\
\hline
\end{tabular}

Origin and name/pedigree of the wheat genotypes used in this work. Numbers correspond to the ID numbers of the Soil-Plant-Water Relations Laboratory at University of Chile 


\section{Determinación del ajuste osmótico (AO)}

Con la información experimental de CRA y $\psi$ s se calculó el ajuste osmótico por tres métodos comúnmente usados en la literatura. El primero (método 1) estimó el AO como la diferencia de $\psi$ sh para cada genotipo entre plantas regadas y plantas estresadas $(\Delta \psi \mathrm{sh})$. La estimación se hizo en cada una de 10 ocasiones de muestreo y se obtuvo un promedio de AO para cada genotipo. El segundo método de cálculo (método 2), descrito por Morgan (1992), usa valores de potencial de solutos sin hidratar la hoja ( $\psi \mathrm{s})$ y sólo necesita un ensayo sin riego. Considera que el valor de $\psi s$ puede disminuir por dos factores, por acumulación activa de solutos al interior de la célula, lo que indica ajuste osmótico, y/o por deshidratación de los tejidos, en que la disminución de $\psi \mathrm{s}$ se debe a un aumento de concentración de solutos por salida de agua. Entonces, el ajuste osmótico corresponde a la diferencia de dos regresiones, una que representa la acumulación de solutos en los tejidos de la planta y otra que representa el efecto de concentración debido a la pérdida de agua.

El valor de AO se obtiene a partir de la regresión entre $\psi$ s y CRA utilizando todas las observaciones realizadas. Paralelamente se estima $\psi \mathrm{s}_{0}$, que corresponde al efecto de concentración por la pérdida de agua, y que se calcula para cada valor de CRA de acuerdo a la formula:

$$
\psi \mathrm{s}_{0}=\psi \mathrm{s}_{\mathrm{i}}\left(\mathrm{CRA}_{\mathrm{i}} / \mathrm{CRA}\right)
$$

donde $\psi s_{i}$ es el $\psi s$ inicial y $\mathrm{CRA}_{\mathrm{i}}$ es el CRA inicial, ambos fueron medidos en plantas bien hidratadas el día 77 después de emergencia. Luego se hace una regresión lineal de $\psi \mathrm{s}_{0}$ sobre CRA. El AO corresponderá a la diferencia entre los valores de $\psi$ s y $\psi s_{0}$ a un valor de CRA de $70 \%$ (turgor cero en plantas de trigo (Morgan 1992)), debido a que normalmente ys es mayor que $\mathrm{ys}_{0}$, esta diferencia es negativa, sin embargo, el AO se expresa en forma positiva. Por conveniencia, las regresiones pueden ser logarítmicas para obtener un mejor ajuste, pero en este caso no fue necesario, no observándose efecto alguno en los resultados al usar una transformación logarítmica.

El tercer método (método 3 ) de cálculo de ajuste osmótico usa la diferencia de $\psi s 100$ (ecuación 3 ) entre plantas que han pasado por un período de estrés hídrico y plantas regadas (Wilson et al. 1979). Esta diferencia se calculó en cada uno de los muestreos y se obtuvo un promedio de AO para cada genotipo.

\section{Análisis de la información fisiológica}

Una vez realizados los análisis de varianza y con el fin de disminuir el efecto ambiental sobre los parámetros hídricos foliares el análisis de la información se hizo utilizando un modelo de regresión lineal propuesto por Eberhart \& Russell (1966). El modelo hace una regresión del valor del genotipo sobre el valor medio de todos los genotipos en cada medioambiente (oportunidad de muestreo en este caso) y estima un coeficiente de regresión y la desviación de la regresión. Así, los valores para cada parámetro hídrico foliar de cada genotipo se ajustaron a un modelo lineal en el que la variable independiente fue la media del parámetro para todos los genotipos en cada oportunidad de muestreo y la variable dependiente la observación correspondiente a un genotipo dado en esa oportunidad de muestreo. Esto se hizo para cada uno de los parámetros foliares de cada genotipo incluyendo las 10 ocasiones de muestreo (cada una considerada como un medioambiente diferente). El comportamiento de cada parámetro hídrico en cada genotipo se puede conocer por la media y la pendiente de la regresión, removiendo así el ruido ambiental asociado a la regresión.

La selección de genotipos se realizó usando el programa de selección ALFA (Barreto et al. 1993), que permite seleccionar genotipos considerando varios parámetros a la vez, asignándoles a cada uno una intensidad de acuerdo a la importancia que tengan respecto a los demás, y una meta de selección de acuerdo a la desviación estándar del parámetro respecto de la media de la población.

\section{Medición del rendimiento y sus componentes}

Para determinar el rendimiento se cosechó a ras de suelo cuatro hileras de $1 \mathrm{~m}$ de largo al centro de cada parcela, se separaron 50 tallos con espigas que se secaron en una estufa de aire forzado por $48 \mathrm{~h}$ a $70{ }^{\circ} \mathrm{C}$. El resto del material se pesó y trilló en una máquina trilladora estacionaria. Los granos fueron pesados y se determinó su porcentaje de humedad. A partir de estos datos e información fenológica se calculó el rendimiento, la biomasa aérea, el peso seco de 1.000 granos, número de espigas por $\mathrm{m}^{2}$, número de granos por $\mathrm{m}^{2}$, granos por espiga, masa de grano (g) por espiga, tasa de producción de grano y tasa de producción de biomasa. El rendimiento en grano se corrigió por el contenido de humedad y se expresa a un $0 \%$ de humedad. 


\section{RESULTADOS}

\section{Parámetros hídricos}

El contenido de agua en el perfil del suelo en los primeros $60 \mathrm{~cm}$ fluctuó entre CC y $50 \%$ de humedad aprovechable en el ensayo regado y entre CC y PMP en el ensayo sin riego. Este último ensayo llegó a PMP el día 115 después de la emergencia (Fig. 1). Dos lluvias cayeron los días 93 y 95 después de emergencia, que sumaron $65,2 \mathrm{~mm}$, hicieron aumentar el contenido hídrico del suelo en ambos ensayos. La Fig. 2 muestra la evolución de los promedios de CRA y de $\psi$ sh para todos los genotipos en las 10 ocasiones de muestreo. El CRA y $\psi$ sh disminuyeron a medida que aumentó el estrés hídrico. El $\psi$ sh promedio de los genotipos en el ensayo sin riego disminuyó en 0,64 $\mathrm{MPa}$, de $-1,17 \mathrm{MPa}$ a $-1,81 \mathrm{MPa}$, a medida que el contenido de humedad del suelo varió entre CC y PMP. El CRA medio de los genotipos en el ensayo sin riego disminuyó de 90 a $64 \%$ y el ajuste osmótico tuvo un máximo medio de 0,54 MPa el día 100 después de emergencia (Fig. 2B, diferencia entre las dos curvas de $\psi$ sh), valor que se observó antes que el suelo llegara a PMP. Las lluvias caídas provocaron aumentos de $\psi$ sh y CRA lo que redujo la diferencia entre ambos ensayos, además provocó una disminución del AO.

El análisis combinado de varianza señaló que los valores de CRA y $\psi$ sh tuvieron efectos altamente significativos de riego (ensayo), muestreo, repetición (dentro de muestreo y riego) y de genotipo (Tabla 2) siendo el aporte de genotipo de $1,6 \%$ de la suma de cuadrados para el caso de CRA y de $6,8 \%$ de la suma de cuadrados para $\psi$ sh. La interacción riego x muestreo (fecha de muestreo) fue muy significativa en el análisis combinado de varianza de ambos parámetros hídricos, reflejando el hecho que tanto el CRA como el $\psi$ sh no variaron significativamente entre muestreos en el ensayo regado, pero si lo hicieron en el ensayo no regado (Fig. 2). La interacción riego $\mathrm{x}$ genotipo fue significativa en el caso de CRA, evidenciando la existencia de diferencias en el comportamiento de CRA de los genotipos ante el déficit hídrico (Fig. 3). Al analizar el porcentaje de la suma de cuadrados de CRA y $\psi$ sh en los ensayos regado y no regado (Tabla 3 ), el CRA varió entre muestreos sólo cuando éstos fueron sometidos a déficit hídrico, situación en la

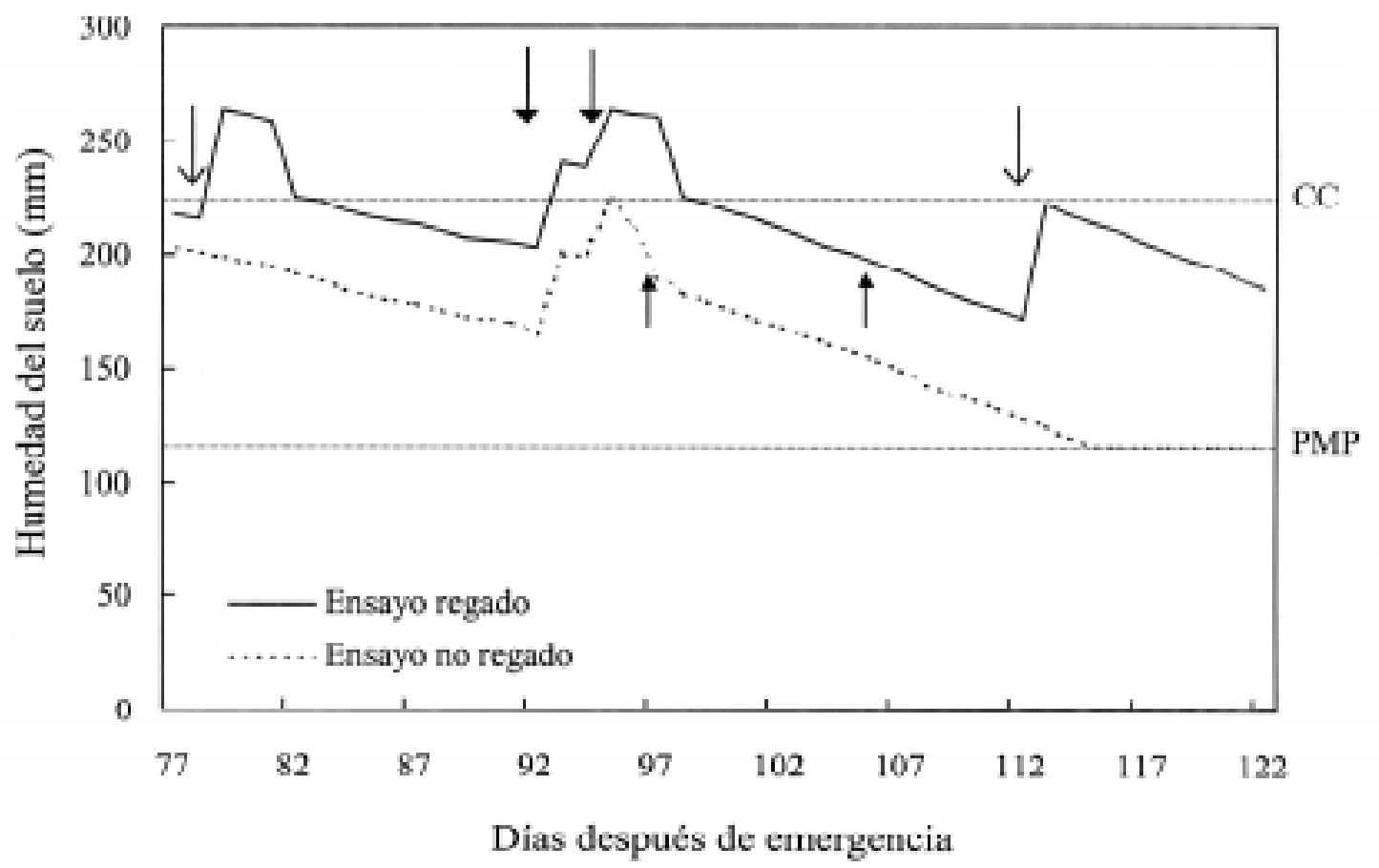

Fig. 1: Evolución del contenido hídrico del suelo en los ensayos de riego y secano durante el período de observaciones: $(\downarrow)$ indican los riegos aplicados en el ensayo regado; $(\downarrow)$ lluvias que cayeron sobre ambos ensayos; $(\uparrow)$ fecha de floración en ambos ensayos.

Soil water content in the irrigated and non irrigated trials during the measurement period: $(\downarrow)$ indicate irrigations over the irrigated trial; $(\downarrow)$ rainfall events; $(\uparrow)$ flowering date in both trials. 


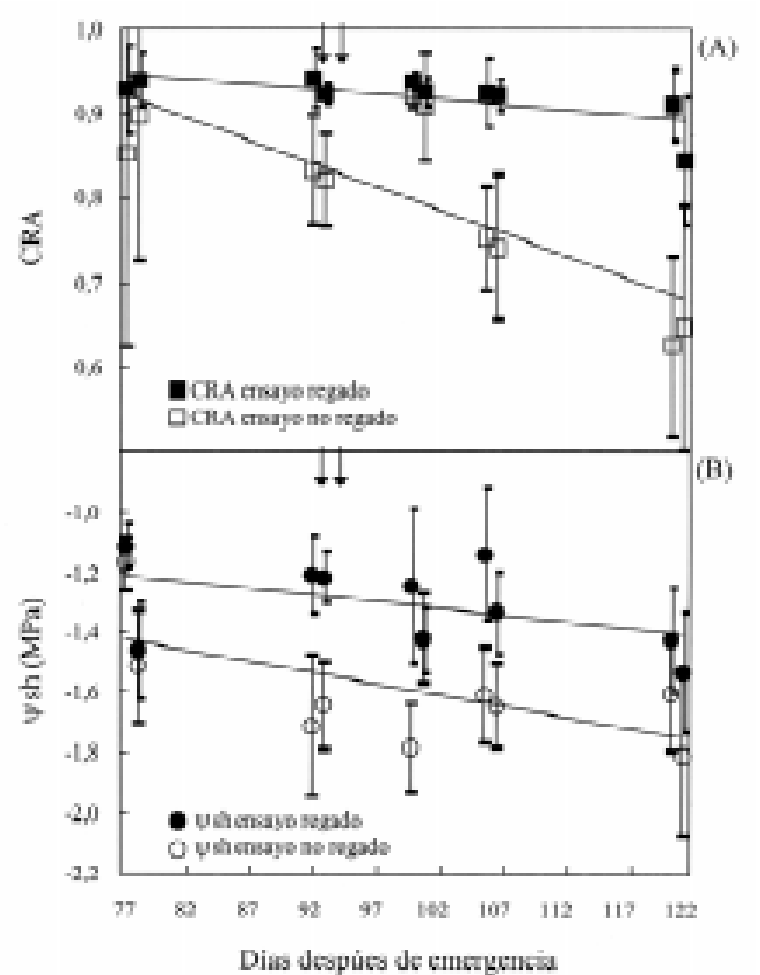

Fig. 2: Evolución del CRA (A) y de $\psi$ sh (B) en los ensayos regado y no regado. Los valores corresponden al promedio de los 31 genotipos en cada muestreo, las barras indican el error estándar de cada media y $(\downarrow)$ indican las lluvias que cayeron sobre ambos ensayos. Se incluyen las líneas de regresión lineal de los parámetros analizados en cada ensayo.

Values of RWC (A) and $\psi$ sh (B) in the irrigated and non irrigated trials. The values are the mean of 31 genotypes for each sampling date. Bars indicate the error standard error of each mean; $(\downarrow)$ indicate rainfall events and the lines indicate the regression lines through the data points.

que también existió variación entre los genotipos y la interacción muestreo x genotipo fue significativa. El $\psi$ sh varió significativamente con genotipo en los ensayos regado y no regado, la interacción riego x genotipo para $\psi$ sh (Tabla 2) no fue significativa, señalando que las diferencias entre genotipos en cuanto a $\psi$ sh se manifiestan independientemente del contenido hídrico del suelo. La correlación de $\psi$ sh entre ensayos fue de $0,56(\mathrm{P} \leq 0,001)$.

El ajuste osmótico obtenido por diferencia promedio de $\psi$ sh entre los ensayos regados y no regados no tuvo efectos de genotipo ni de muestreo (método 1); tampoco se observaron diferencias entre genotipos en los valores de ajuste osmótico calculados a partir de los valores de $\psi$ s 100 (método 3). Los valores promedio de ajuste osmótico, estimados por los 3 métodos de cálculo, para los 31 genotipos de trigo fueron de $0,28 \mathrm{MPa}( \pm 0,08)$ para el método $1 ; 1,48 \mathrm{MPa}( \pm 0,47)$ para el método 2 (calculado para cada genotipo de acuerdo a la figura 4$)$ y $0,36 \mathrm{MPa}( \pm 0,1)$ para el método 3 .

Metodología de análisis de los parámetros hídricos foliares

Los valores medios de los parámetros $\psi$ sh, CRA y AO fueron graficados sobre la pendiente de la

\section{TABLA 2}

Porcentaje de la suma de cuadrados de CRA y $\psi$ sh atribuibles a diferentes factores según ANDEVA combinado sobre ensayos de riego y fechas de muestreo

Percentage of the sum of squares of RWC and $\psi$ sh attributable to various factors according to a combined

ANOVA over irrigation trials and sampling dates

\begin{tabular}{lcr}
\hline & \multicolumn{2}{c}{ Suma de cuadrados $(\%)$} \\
& CRA & \multicolumn{1}{c}{$\psi$ sh } \\
\hline Riego (R) & $29,0^{* * *}$ & $32,7 * * *$ \\
Muestreo (M) & $37,7 * * *$ & $13,1 * * *$ \\
RM & $17,2 * * *$ & $9,1 * * *$ \\
Repetición (MR) & $1,7 * * *$ & $18,7 * * *$ \\
Genotipo (G) & $1,6^{* *}$ & $6,8 * * *$ \\
RG & $1,7 *$ & $2,1 \mathrm{~ns}$ \\
MG & 5,2 n.s. & $9,0 \mathrm{~ns}$ \\
RMG & $5,8 *$ & $8,2 \mathrm{~ns}$ \\
\hline
\end{tabular}

$(*)=\mathrm{P} \leq 0,05 ;(* *)=\mathrm{P} \leq 0,01 ;(* * *)=\mathrm{P} \leq 0,001 ;$

$(\mathrm{ns})=$ estadísticamente no significativo

TABLA 3

Porcentaje de la suma de cuadrados de CRA y $\psi$ sh atribuibles a genotipo, fecha de muestreo e interacción según ANDEVA combinado sobre fechas de muestreo para los ensayos regado y no regado

Percentage of the sum of squares of RWC and $\psi$ sh attributable to genotype, sampling date and genotype $\mathrm{x}$ sampling date according to a combined ANOVA over sampling dates for the irrigated and non irrigated trials

\begin{tabular}{lrlll}
\hline & \multicolumn{2}{c}{ No regado } & \multicolumn{2}{c}{ Regado } \\
& CRA & $\psi$ ssh & CRA & $\psi$ \%sh \\
\hline Muestreo & $82,0 * * *$ & $48,4 \mathrm{~ns}$ & $46,2 \mathrm{~ns}$ & $42,0 \mathrm{~ns}$ \\
Genotipo & $4,3 * * *$ & $19,6 * * *$ & $13,0 \mathrm{~ns}$ & $16,8 * * *$ \\
MG & $13,5 * * *$ & $32,0 \mathrm{~ns}$ & $40,7 \mathrm{~ns}$ & $41,2 \mathrm{~ns}$ \\
\hline
\end{tabular}

$(*)=\mathrm{P} \leq 0,05 ;(* *)=\mathrm{P} \leq 0,01 ;(* * *)=\mathrm{P} \leq 0,001 ;$

(ns) = estadísticamente no significativo 


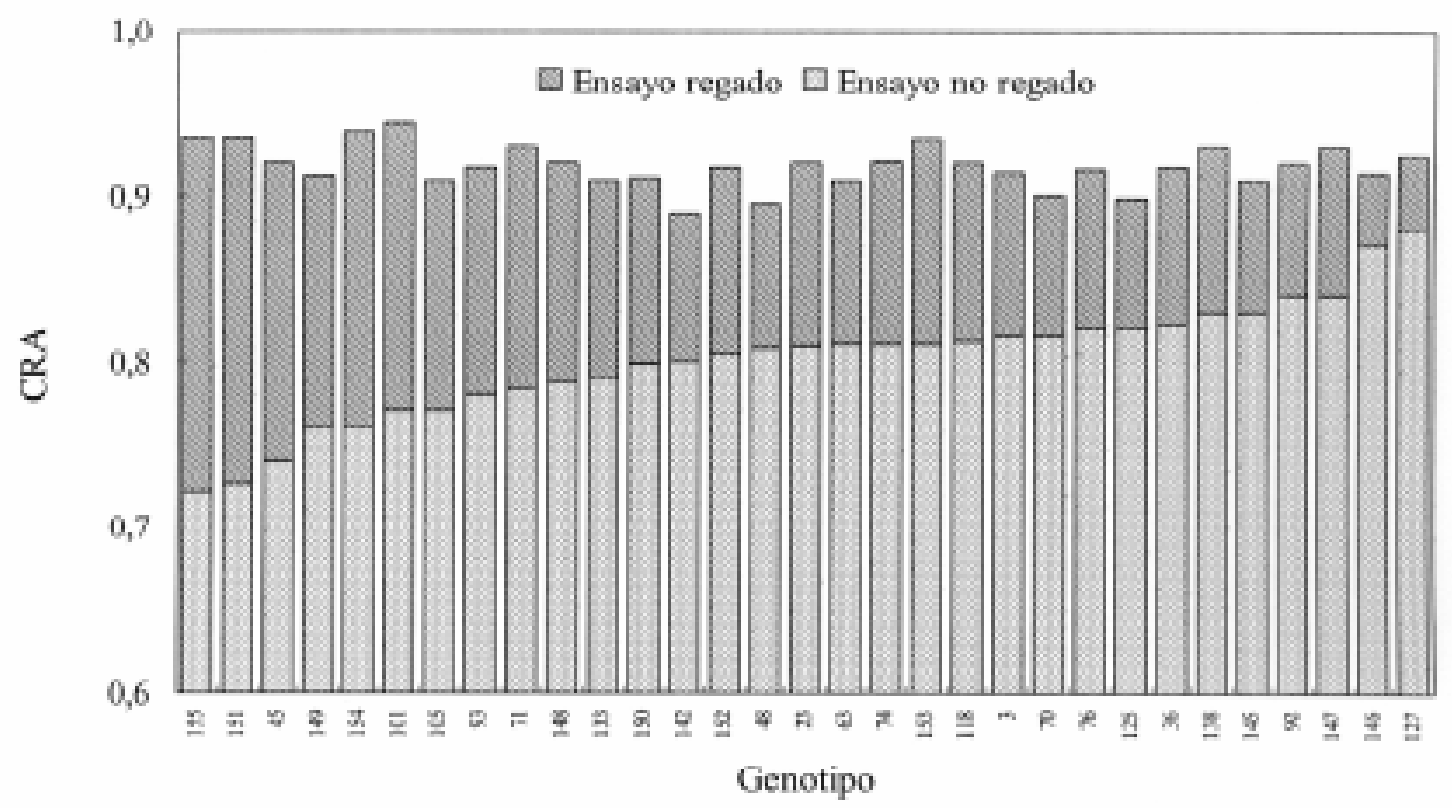

Fig. 3: Valores de promedio de CRA para cada genotipo en los ensayos regado y no regado.

Mean values of RWC of each genotype in the irrigated and non irrigated trials.

regresión del parámetro de cada genotipo (Fig. 5, 6 y 7 , respectivamente). La zona achurada de la Fig. 5 encierra a los genotipos de pendiente de భsh mayor a uno y que están una desviación estándar sobre la media general; en la Fig. 6 la zona achurada encierra los genotipos de pendiente de CRA menor a uno y cuyo promedio es mayor en una desviación estándar a la media general; y en la Fig. 7 se destacan los genotipos de pendiente de AO mayor a uno y que están sobre la media más una desviación estándar.

El promedio general de $\psi$ sh se correlacionó negativamente con el promedio general de CRA (Tabla 4). También hubo correlaciones negativas entre el promedio de $\psi$ sh y la pendiente de $\psi$ sh, entre el promedio de CRA y la pendiente de CRA y entre el promedio de $\psi$ sh y el promedio de AO.

Se hicieron dos selecciones de genotipos, la primera consideró la pendiente y el promedio de $\psi$ sh, la pendiente y el promedio de CRA, y la pendiente y el promedio de AO (método 1); la segunda selección consideró la pendiente y el promedio de $\psi \mathrm{sh}$, y la pendiente y el promedio de CRA. Estas selecciones se ordenaron de menor a mayor. Paralelamente los genotipos se ordenaron de menor a mayor de acuerdo al valor de AO, lo que se realizó con los valores de los tres métodos de estimación por separado. Luego se le asignó un número de orden a cada genotipo y se correlacionaron los distintos ordenes (Tabla 5). El orden establecido por el método de calculo de AO por las diferencias medias de $\psi$ sh (método 1 ) tuvo una correlación altamente significativa con el orden establecido por las pendientes de $\psi$ sh, CRA y AO y con el orden establecido por la pendiente y el promedio de $\psi$ sh y CRA, situación que se repitió con el método 3 . El orden establecido por el método 2 se correlacionó con el orden

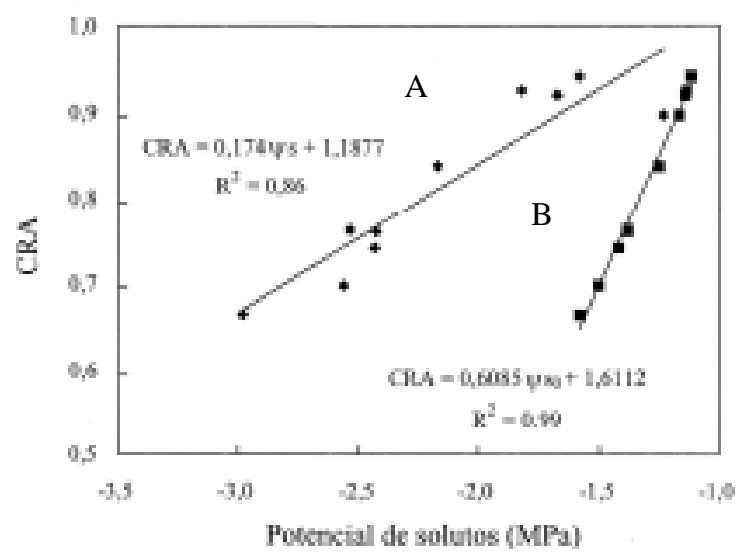

Fig. 4: Ejemplo de regresiones realizadas para calcular AO según el método de Morgan (1992) para el genotipo 3: (A) valores de $\psi$ s sobre CRA; (B) valores de $\psi \mathrm{s}_{0}$ sobre CRA. El AO es estimado como la diferencia entre ambas regresiones a un CRA de $70 \%$.

Example of regressions used to calculate osmotic adjustment according to Morgan (1992) for genotype 3: (A) values of $\psi$ s over RWC; (B) values of $\psi s_{0}$ over RWC. The $\mathrm{OA}$ is estimated as the difference between the two regressions at a RWC of $70 \%$. 


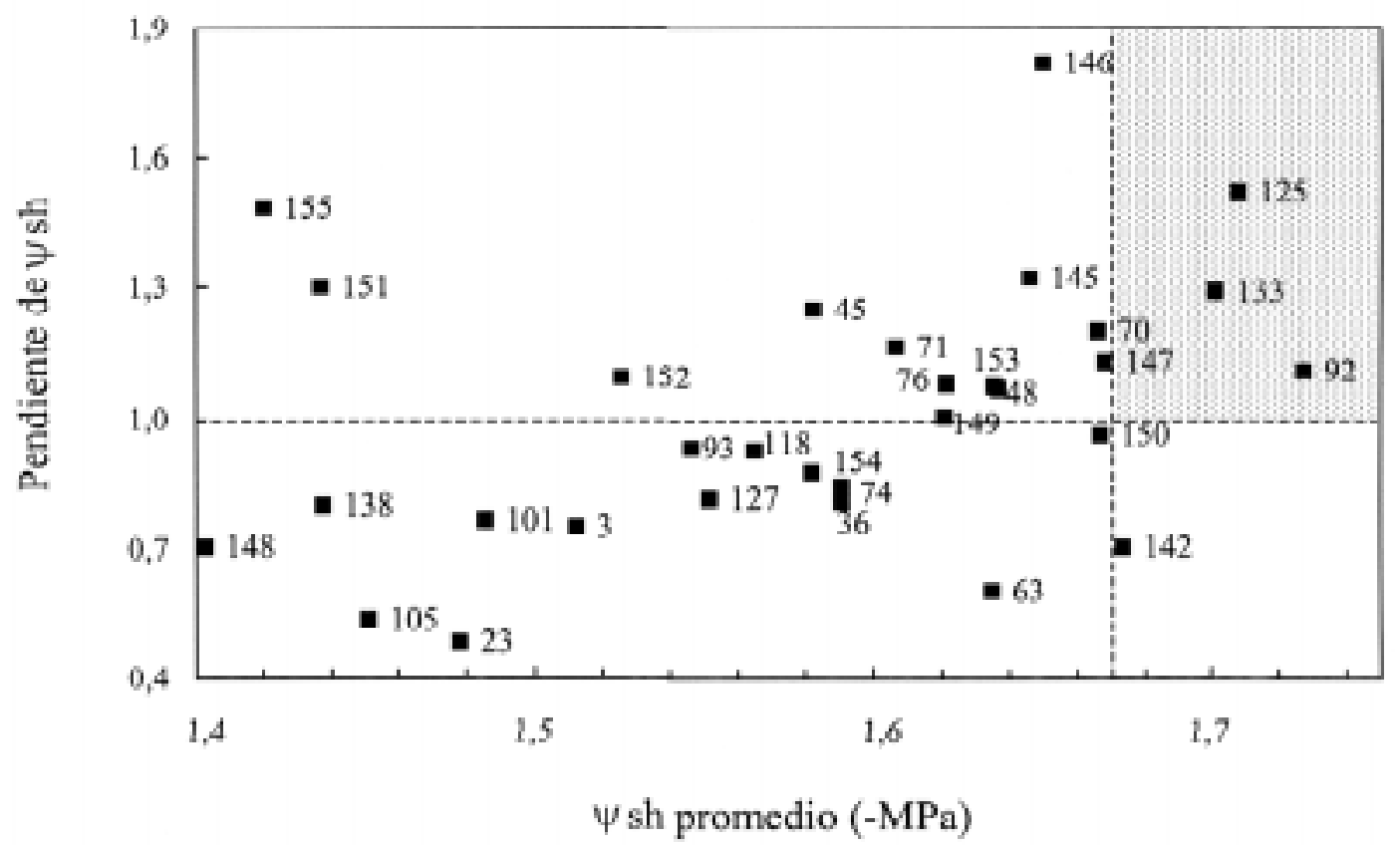

Fig. 5: Selección de genotipos de acuerdo a su pendiente y promedio de $\psi$ sh, medido en el ensayo no regado. El área achurada señala los genotipos que se ubican una desviación estándar sobre el promedio general.

Selection of genotypes according to their slope and mean $\psi$ sh as measured in the non irrigated trial. The shaded area shows the genotypes one standard deviation above the grand mean.

establecido por el promedio y las pendientes de $\psi s h$, CRA y AO.

Considerando las pendientes y promedios de $\psi$ sh, CRA y AO se realizó una selección de tres genotipos, de acuerdo a los requisitos de pendiente y promedio impuestos en las Fig. 5, 6 y 7, resultando elegidos los genotipos 147, 133 y 125 . También se escogieron los genotipos 105,148 y 101 con características contrarias a las establecidas, es decir baja pendiente y alto promedio de $\psi$ sh, alta pendiente y bajo promedio de CRA y baja pendiente y bajo promedio de AO. En la Fig. 8A y 8B, se aprecian curvas de regresión de $\psi$ sh y CRA de los genotipos seleccionados. Se observa la diferencia en las pendientes de las curvas de los genotipos, alta pendiente de $\psi s h$, pero baja pendiente de CRA para los genotipos que ajustan osmóticamente, que contrasta con los genotipos 105, 148 y 101, en que las pendientes de las curvas son opuestas, y en los que el ajuste osmótico es menor.

TABLA 4

Correlaciones entre $\psi$ sh promedio, CRA promedio, AO promedio, pendiente de $\psi$ sh, pendiente de CRA y pendiente de AO

Correlations among mean $\psi$ sh, mean RWC, mean OA, slope of $\psi$ sh, slope of RWC and slope of OA

\begin{tabular}{|c|c|c|c|c|c|}
\hline & CRA $\bar{x}$ & $\mathrm{AO} \overline{\mathrm{x}}$ & $\begin{array}{l}\text { Pendiente } \\
\psi \text { sh }\end{array}$ & $\begin{array}{c}\text { Pendiente } \\
\text { CRA }\end{array}$ & $\begin{array}{c}\text { Pendiente } \\
\text { AO }\end{array}$ \\
\hline$\psi \operatorname{sh} x$ & $-0,4415 *$ & $-0,5731 * * *$ & $-0,3724 *$ & 0,3411 & $-0,0543$ \\
\hline CRA $\bar{x}$ & 1,0000 & 0,1838 & 0,0072 & $-0,4648 * *$ & $-0,2784$ \\
\hline $\mathrm{AO} \overline{\mathrm{x}}$ & & 1,0000 & 0,2585 & $-0,1882$ & 0,2476 \\
\hline Pendiente $\psi$ sh & & & 1,0000 & $-0,0931$ & $0,4029 *$ \\
\hline Pendiente CRA & & & & 1,0000 & 0,2666 \\
\hline Pendiente AO & & & & & 1,0000 \\
\hline
\end{tabular}

$(*)=\mathrm{P} \leq 0,05 ;(* *)=\mathrm{P} \leq 0,01 ;(* * *)=\mathrm{P} \leq 0,001$ 


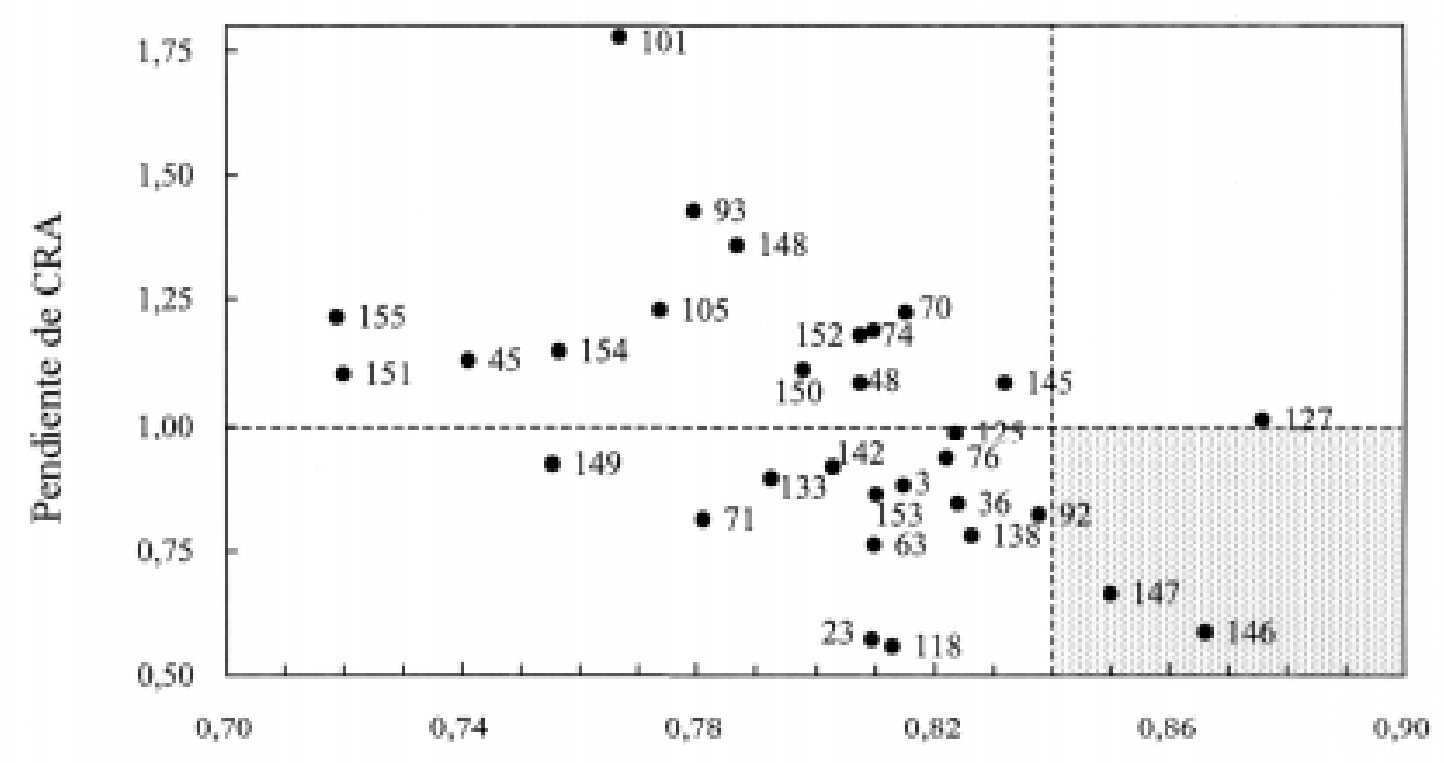

\section{CRA promedio (\%)}

Fig. 6: Selección de genotipos de acuerdo a su pendiente y promedio de CRA, medido en el ensayo no regado. El área achurada señala los genotipos que se ubican una desviación estándar sobre el promedio general.

Selection of genotypes according to the slope and mean RWC as measured in the non irrigated trial. The shaded area shows the genotypes one standard deviation above the grand mean.

\section{Rendimiento}

El rendimiento bajo sequía fue aproximadamente el $50 \%$ del rendimiento bajo riego (3.609 y 6.917 $\mathrm{kg} \mathrm{ha}^{-1}$, respectivamente). De los componentes de rendimiento el número de granos $/ \mathrm{m}^{2}$ fue el que mostró la mayor disminución (37\%). El rendimiento bajo sequía fue explicado en proporción similar por el número de granos $\mathrm{m}^{-2} \mathrm{y}$ el peso de los granos, a la vez, los granos $\mathrm{m}^{-2}$ dependieron en un $43 \%$ de las espigas $\mathrm{m}^{-2}$ y en un $56 \%$ de los granos espiga $^{-1}$ (Tabla 6).

El análisis combinado de varianza de los ensayos regado y no regado mostró efectos muy significativos de riego y genotipo sobre el rendimiento. Al factor riego (ensayo) es atribuible un $82 \%$ de la suma de cuadrados y al factor genotipo, un $13 \%$. La interacción riego $\mathrm{x}$ genotipo no fue significativa, a pesar de que los análisis de varianza simples para cada ensayo mostraron que en el ensayo regado no hubo diferencias significativas para rendimiento entre genotipos y que éstas fueron muy significativas en el ensayo no regado.

En el análisis de la relación de los parámetros hídricos foliares con el rendimiento se incluyó el rendimiento bajo estrés hídrico de cada genotipo corregido por los días a antesis de cada uno, ya que la floración temprana constituye un mecanismo de escape a la sequía, lo que significa que genotipos con fecha de floración temprana sufrirán en menor medida los efectos del estrés hídrico. Luego se correlacionaron los parámetros hídricos foliares con el rendimiento y otros parámetros asociados como biomasa y parámetros derivados del rendimiento de cada genotipo como el Índice

\section{TABLA 5}

Correlación entre los órdenes ascendentes de genotipos seleccionados por diferentes parámetros hídricos

Rank correlation of ascending order of genotypes selected by various leaf water parameters

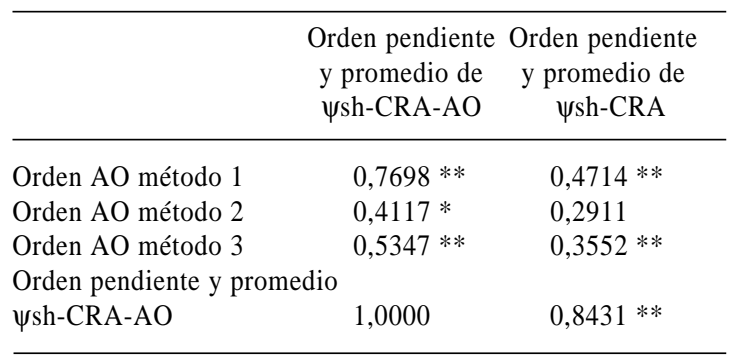

$(*)=\mathrm{P} \leq 0,05 ;(* *)=\mathrm{P} \leq 0,01 ;(* * *)=\mathrm{P} \leq 0,001$ 
TABLA 6

Contribución al rendimiento de los diferentes componentes. Estimación realizada por una regresión múltiple realizada paso a paso

Contribution of the various yield components to grain yield as calculated by stepwise regression

\begin{tabular}{lcc}
\hline & \multicolumn{2}{c}{ Delta $\mathrm{R}^{2 *}$} \\
& $\begin{array}{c}\text { Rendimiento } \\
\text { ensayo } \\
\text { no regado }\end{array}$ & $\begin{array}{c}\text { Rendimiento } \\
\text { ensayo } \\
\text { regado }\end{array}$ \\
\hline PS 1.000 granos & 0,488 & 0,596 \\
Granos m-2 & 0,498 & 0,389 \\
Espigas m$^{-2}$ & 0,428 & 0,691 \\
Granos espiga-1 $^{-2}$ & 0,560 & 0,258 \\
\hline
\end{tabular}

* Proporción de la suma de cuadrados debida a regresión explicada por cada variable

de Respuesta a la Sequía, DRI (Bidinger et al. 1987), y la pendiente de la relación entre rendimiento y el rendimiento medio de todos los genotipos $(\mathrm{P})$. Se encontraron cuatro correlaciones significativas entre parámetros hídricos y rendimiento: rendimiento bajo estrés versus pendiente $\psi \mathrm{sh}(\mathrm{r}=-0,44 ; \mathrm{P} \leq 0,01)$; rendimiento bajo estrés corregido por días a antesis vs pendiente $\psi \operatorname{sh}(\mathrm{r}=-0,53 ; \mathrm{P} \leq 0,01)$ y versus ajuste osmótico calculado de acuerdo a Morgan (1992) ( $r=-0,38$; $\mathrm{P} \leq 0,05)$; y $\mathrm{P}$ versus la pendiente de $\mathrm{AO}(\mathrm{r}=0,36$; $\mathrm{P} \leq 0,05)$.

\section{DISCUSIÓN}

Variabilidad de los parámetros hídricos foliares y justificación de la metodología de análisis

La alta proporción de la suma de cuadrados correspondiente al factor muestreo en la variación de los parámetros hídricos foliares dentro de un ensayo determinado (40-80\%, Tabla 3), asociada a una baja interacción muestreo x genotipo (MG), junto a una nula correlación entre repeticiones muestreadas en días consecutivos de los parámetros $\psi$ sh y CRA puso en evidencia un alto efecto ambiental en la determinación de estos parámetros. Por lo anterior se exploró la regresión lineal como otra forma de análisis de la información obtenida.

En el caso de $\psi$ sh, lo esperado en una planta de trigo de mayor resistencia a sequía es que disminuya su valor frente al estrés hídrico más allá del valor correspondiente a la disminución asociada

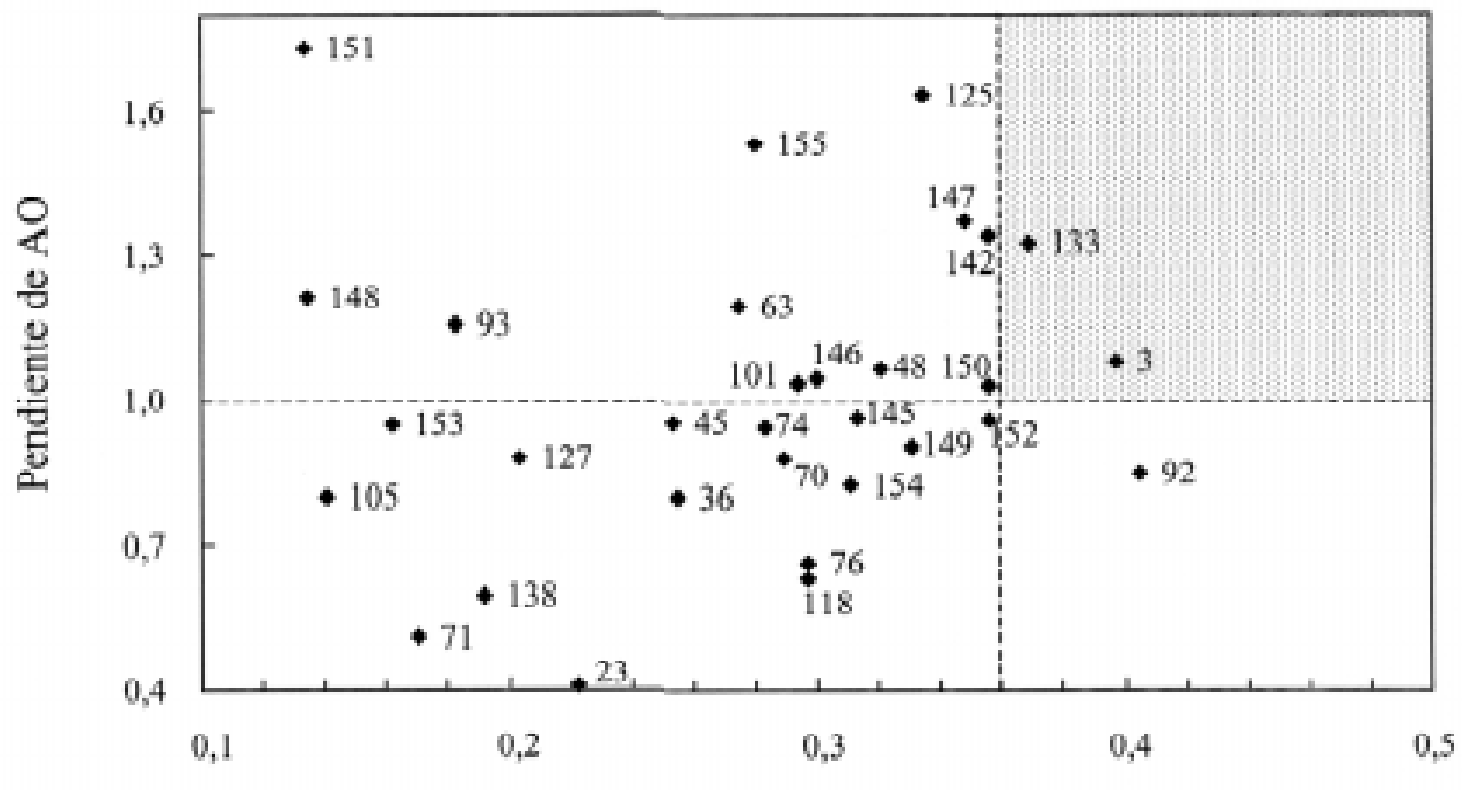

AO promedio $(\mathrm{MPa})$

Fig. 7: Selección de genotipos de acuerdo a su pendiente y promedio de AO, medido en el ensayo no regado. El área achurada señala los genotipos que se ubican una desviación estándar sobre el promedio general.

Selection of genotypes according to the slope and mean OA of the non irrigated trial. The shaded area shows the genotypes one standard deviation above the grand mean. 

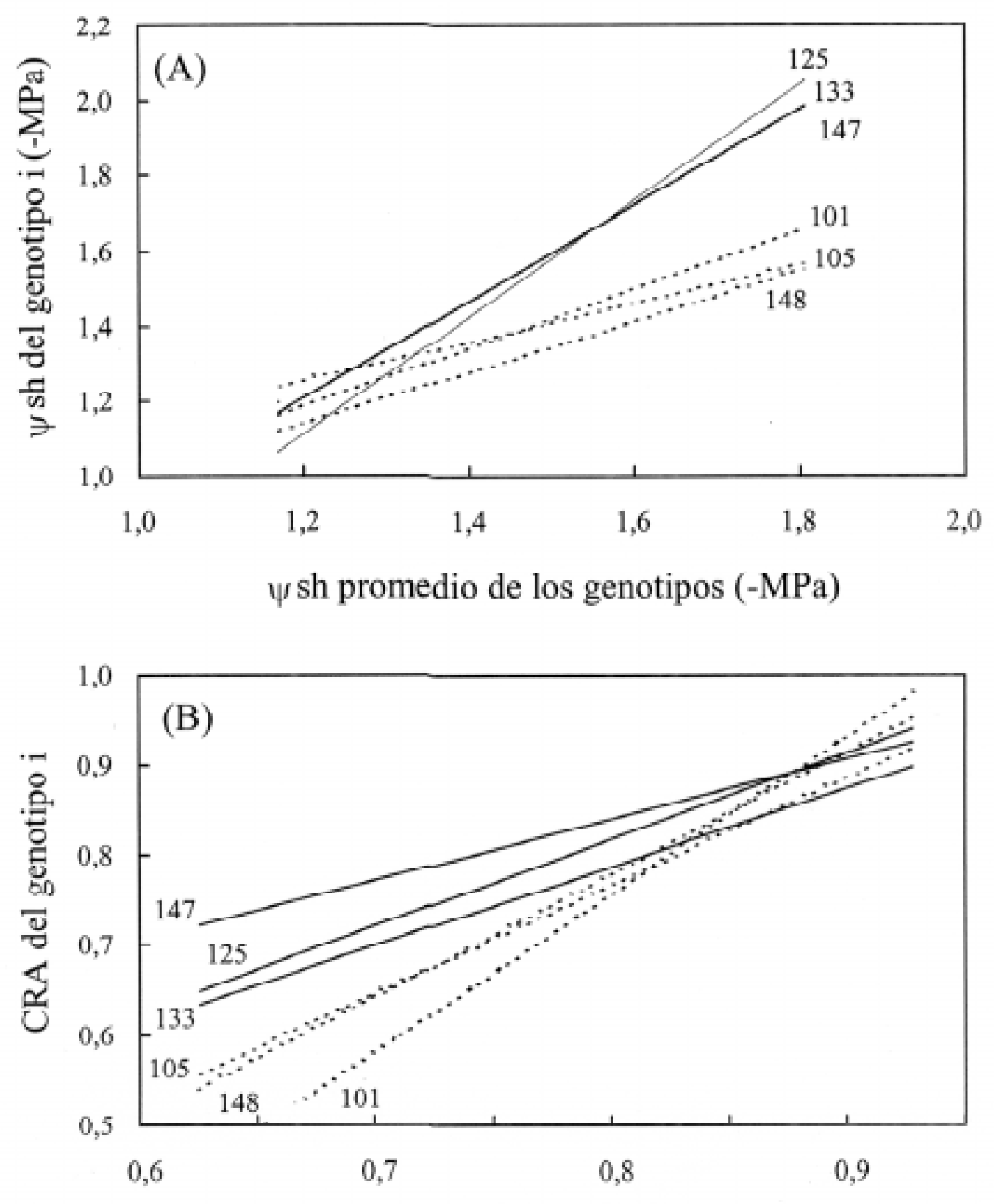

CRA promedio de los genotipos

Fig. 8: Curvas de regresión de los genotipos seleccionados por alta pendiente y alto promedio de $\psi$ sh y baja pendiente y alto promedio de CRA (147, 133 y 125, línea continua en A y B) y de los genotipos seleccionados por baja pendiente y bajo promedio de $\psi$ sh y alta pendiente y bajo promedio de CRA (105, 148 y 101, línea segmentada en A y B).

Regression curves of the genotypes selected for high slope and high mean $\psi$ sh and low slope and high values of RWC (147, 133 and 125, continuous line in parts A and B) and of the genotypes selected for low values of slope and low mean $\psi$ sh and high values of slope and low values of mean RWC (105, 148 and 101, dashed line parts A and B). 
al aumento de concentración por deshidratación (Hsiao et al. 1976, Bajii et al. 2000). De acuerdo a esto, si se hace la regresión de los valores de $\psi$ sh de un genotipo determinado sobre el promedio de $\psi$ sh de toda la población de genotipos analizada en cada una de las ocasiones de muestreo, lo esperado para un genotipo que aumenta la concentración de solutos al interior de las células sobre el promedio de los genotipos, sería una pendiente mayor a uno. Entonces, un criterio para seleccionar genotipos resistentes a sequía, analizando la pendiente del potencial de solutos y su promedio, sería elegir aquellos de alta pendiente de $\psi$ sh (mayor a 1) y que además tengan un valor absoluto promedio de $\psi$ sh mayor al promedio general más una desviación estándar. La asociación entre ambos se aprecia en la correlación negativa que mostraron (Tabla 4, bajo valor promedio, alta pendiente).

El CRA de un genotipo resistente a la sequía debiera tener valores altos a pesar del avance del estrés hídrico, lo que le permitiría a la planta mantener el turgor ( $\psi \mathrm{p}$ ) (Gesch et al. 1992) y los procesos que de él dependen. Esto implica que al considerar la regresión de los valores de CRA de un genotipo sobre el promedio de todos los genotipos muestreados en un ciclo de déficit hídrico, deberían identificarse aquellos que tengan un valor de pendiente menor a uno, es decir, cuyo CRA sea estable, y el promedio sea mayor al promedio general más una desviación estándar. El promedio y la pendiente de CRA también tuvieron una correlación negativa (Tabla 4), lo que corrobora la asociación entre ambos.

En el caso de AO, lo esperado en un genotipo que ajuste osmóticamente es que vaya aumentando el AO con el avance del estrés hídrico, por lo tanto, el valor de la pendiente que refleja este aumento de $\mathrm{AO}$ es mayor a uno (similar a lo que ocurre con $\psi \mathrm{sh}$ ) y su promedio debería ser mayor al promedio general más una desviación estándar.

En síntesis, los genotipos de mayor resistencia a sequía de acuerdo a los valores de pendiente y promedio de los parámetros hídricos foliares $\psi s h$, CRA y AO son aquellos de alta pendiente y alto valor absoluto promedio de $\psi$ sh, baja pendiente y alto promedio de CRA y alta pendiente y alto promedio de AO.

Las relaciones experimentales entre parámetros hídricos foliares (Tabla 4) señalan que la disminución de $\psi$ sh estuvo asociada a un alto valor de CRA en condiciones de sequía, es decir, la acumulación de solutos en respuesta al déficit hídrico (AO) ayudaría a mantener el CRA a nivel foliar. Lo anterior concuerda con lo señalado por Gesch et al. (1992) y Teulat et al. (1997) y es consistente con los requisitos de selección establecidos.
De acuerdo a la discusión anterior, se desprende que el método más adecuado de determinación de AO será aquel que represente de mejor manera la evolución del $\psi$ sh y de CRA a medida que avanza el estrés hídrico, ya que el AO es un indicador que integra ambos parámetros. Su mejor estimación será aquella que identifique genotipos que presenten la mejor evolución de los parámetros hídricos foliares ante un ciclo de sequía.

Un punto a considerar es la elasticidad de la pared celular que tiene un efecto en las relaciones hídricas de los tejidos (Morgan 1984). Las observaciones realizadas en este trabajo no permiten descartar este efecto, sin embargo, al proponer un método de selección basado en $\psi$ sh, CRA y AO, se buscan genotipos que acumulen solutos en respuesta al estrés hídrico, pero que mantengan valores de CRA altos, lo que restaría importancia a la elasticidad de paredes en estas selecciones. Por otra parte, Wright et al. (1983) señalan que las diferencias varietales en AO se manifiestan independientemente de algún cambio en el modulo de elasticidad.

\section{Selección de genotipos}

El método que más se acerca al orden de los genotipos establecido por la combinación $\psi s h$, CRA y AO sin tener elementos de autocorrelación es el orden de AO determinado por los valores medios de $\psi$ sh. Esto indica que se puede seleccionar genotipos que ajusten osmóticamente usando los criterios establecidos para las pendientes y los promedios de los parámetros hídricos foliares $\psi$ sh, CRA y AO, lo que representa una alternativa de análisis de estos parámetros cuando, debido a su fuerte interacción con el medioambiente, no se pueden realizar los análisis estadísticos tradicionales. Entonces, considerar las repeticiones de un genotipo como observaciones independientes y ajustarlas a un modelo lineal permite remover parte del ruido ambiental que afecta a estos parámetros.

El orden de los genotipos seleccionados de acuerdo a las pendientes y los promedios de $\psi \mathrm{sh}$, CRA y AO también se correlacionó significativamente $(P \leq 0,01)$ con el orden de genotipos que considera la pendiente y promedio de $\psi$ sh y CRA (Tabla 5). Esto sugiere la posibilidad de seleccionar genotipos que ajusten osmóticamente en sólo un ensayo, sin riego, prescindiendo del ensayo regado, similar a lo descrito por Morgan (1983, 1992, 1995), lo que disminuye a la mitad el tamaño del ensayo y el número de observaciones. 


\section{Acumulación de solutos y rendimiento}

La pendiente de $\psi$ sh se correlacionó negativamente con el rendimiento bajo estrés y con el rendimiento bajo estrés corregido por días a antesis. Esta pendiente es un indicador de la tasa de acumulación de solutos, por lo que una alta tasa de acumulación de solutos permite a la planta realizar ajuste osmótico y sobrevivir a la sequía, pero no contribuyó al rendimiento. Genotipos de baja tasa de acumulación de solutos tienen mayor rendimiento bajo sequía. Esta tendencia negativa se mantuvo con los componentes del rendimiento gramos de grano por espiga, tasa de producción de grano y tasa de producción de biomasa.

Babu et al. (1999) señalan que el aporte positivo del ajuste osmótico al rendimiento está condicionado al largo del período de desecamiento del suelo. La tasa de estrés está determinada por la temperatura y la demanda evapotranspirativa (Babu et al. 1999). Jones \& Rawson (1979) aplicaron distintas tasas de estrés a plantas de sorgo y establecieron que se produce AO a tasas bajas y moderadas de estrés $\left(0,15\right.$ y $0,7 \mathrm{MPa} \operatorname{dia}^{-1}$ de disminución de potencial hídrico foliar), pero a tasas altas (1,2 $\left.\mathrm{MPa} \mathrm{dia}^{-1}\right)$ éste no se presenta. En este trabajo la tasa de estrés fue de aproximadamente $0,11 \mathrm{MPa}$ día $^{-1}$ y la evapotranspiración estimada para el cultivo fue de $3,0 \mathrm{~mm} \mathrm{dia}^{-1}$, entre CC y PMP, lo que corresponde a una tasa baja de estrés que se desarrolló en un período de 30 días. Esto coincide con lo observado por Jones \& Rawson (1979). Morgan (1992) señala que se requieren 7 ó 9 días bajo estrés hídrico para que se exprese el $\mathrm{AO}$ en trigo, por lo que en este trabajo la tasa a la que se desarrolló el estrés no debió significar problema para la expresión del AO.

Cabe destacar que la mayoría de las experiencias existentes en la literatura son realizadas bajo condiciones controladas en invernadero, pero a nivel de campo la planta interactúa con varios otros factores. Blum et al. (1999) señalan que el aporte del AO al rendimiento se manifiesta de manera indirecta, como por ejemplo generando un mayor crecimiento de raíces que explorarían un mayor volumen de suelo por agua. En la planta el AO se correlaciona negativamente con la termoestabilidad de las membranas celulares, por lo que la acumulación de solutos protegería varias funciones celulares (Blum \& Pnuel 1990). Los resultados obtenidos en el grupo de genotipos estudiados en este trabajo indican que el AO es un mecanismo que le permite a la planta mantener el nivel hídrico de sus tejidos frente al estrés hídrico, concordando con otros autores (Sen-Gupta \& Berkowitz 1987, Gesch et al. 1992, Khanna \& Chopra 1999), esto probablemente le permita so- brevivir y terminar su ciclo de vida, pero no tener un mayor rendimiento bajo estrés.

\section{CONCLUSIONES}

Existe un alto efecto ambiental asociado a las mediciones de parámetros hídricos foliares lo que dificulta su análisis. Sin embargo, es posible caracterizar genotipos resistentes a sequía basándose en la evolución de los parámetros hídricos foliares en campo. Al obtener la pendiente de la regresión entre la observación de un genotipo y el promedio de todos los genotipos, para los parámetros hídricos foliares $\psi s h$, CRA y AO, y luego usar el valor de la pendiente y el promedio de estos parámetros en selección, se puede determinar genotipos que ajusten osmóticamente, removiendo en parte el ruido ambiental asociado a estos parámetros. El ajuste osmótico que mostraron los genotipos analizados tuvo una correlación negativa con el rendimiento bajo estrés hídrico, lo que estaría indicando que el AO es un mecanismo que permite a la planta sobrevivir al estrés hídrico, pero no tener un rendimiento mayor. Esto descartaría al ajuste osmótico como mecanismo de selección indirecta de genotipos de mayor rendimiento bajo estrés hídrico, en las condiciones de estrés que se presentaron en este trabajo.

\section{AGRADECIMIENTOS}

Este trabajo fue financiado por un proyecto FONDECYT 1990787.

\section{LITERATURA CITADA}

ACEVEDO E (1975) The growth of maize under field conditions as affected by its water relations. Ph.D. Thesis, Department of Water Science and Engineering, University of California, Davis, California, USA. $253 \mathrm{pp}$.

ACEVEDO E, H SILVA \& P SILVA (1998) Tendencias actuales de la investigación en la resistencia al estrés hídrico de las plantas cultivadas. Universidad de Chile, Facultad de Ciencias Agrarias y Forestales, Boletín Técnico (Chile) 49: 1-28.

ACEVEDO E, P SILVA \& H SILVA (1999) Wheat production in mediterranean environments. En: Satorre EH \& GA Slafer (eds) Wheat ecology and physiology of yield determination: 295-331. The Haworth Press, New York, New York, USA.

BABU R, M SAFIULLAH, A BLUM \& H NGUYEN (1999) Comparison of measurement methods of osmotic adjustment in rice cultivars. Crop Science 39: $150-158$. 
BAJII M, S LUTTS \& J KINET (2000) Physiological changes after exposure and recovery from Polyethylene Glycol-induced water deficit in roots and leaves of durum wheat (Triticum durum desf.) cultivars differing in drought resistance. Journal of Plant Physiology 157: 100-108.

BAJII M, S LUTTS \& J KINET (2001) Water deficit effects on solute contribution to osmotic adjustment as a function of leaf age in three durum wheat (Triticum durum desf.) cultivars performing differently in arid conditions. Plant Science 160: 669-681.

BARRETO H, J BOLAÑOS \& H CÓRDOBA (1993) Programa índice de selección. Guía para la operación del software. Centro Regional de Mejoramiento de Maíz y Trigo, Publicación CIMMYT, Distrito Federal, México. 27 pp.

BARRS HD \& PE WEATHERLEY (1962) A reexamination of the relative turgidity technique for estimating water deficits in leaves. Australian Journal of Biological Science 15: 413-428.

BIDINGER F, V MAHALAKSHIMI \& G RAO (1987) Assessment of drought resistance in pearl millet (Pennisetum americanum (L.) Leeke). II Estimation of genotype response to stress. Australian Journal Agricultural Research 38: 49-59.

BLUM A (1989) Osmotic adjustment and growth of barley genotypes under drought stress. Crop Science 29: 230-233.

BLUM A \& C SULIVAN (1986) The comparative drought resistance of landraces of sorghum and millet from dry and humid regions. Annals of Botany 57: 835846.

BLUM A \& Y PNUEL (1990) Physiological attributes associated with drought resistance of wheat cultivars in a mediterranean environment. Australian Journal of Agricultural Research 41: 799-810.

BLUM A, J ZHANG \& H NGUYEN (1999) Consistent differences among wheat cultivars in osmotic adjustment and their relationship to plant production. Field Crops Research 64: 287-291.

COMISIÓN NACIONAL DE RIEGO (1981) Estudio de suelos del Proyecto Maipo. Agrolog Chile Limitada, Santiago, Chile. 10 volumenes.

EBERHART S \& W RUSELL (1966) Stability parameters for comparing varieties. Crop Science 6: 36-40.

EL-HAFID R, D SMITH, M KARROU \& K SAMIR (1998) Physiological responses of spring durum wheat cultivars to early-season drought in a Mediterranean environment. Annals of Botany 81: 363-370.

GESCH R, D KENEFICK \& A KOEPKE (1992) Leaf water adjustment and maintenance in hard red winter wheat. Crop Science 32: 180-186.

GIRMA F \& D KRIEG (1992) Osmotic adjustment in sorghum. Plant Physiology 99: 577-582.

HSIAO T, E ACEVEDO, E FERERES \& D HENDERSON (1976) Stress metabolism. Phylosophical Transactions of the Royal Society of London, Series B 273: 479500 .
JONES M \& H RAWSON (1979) Influence of the rate of development of leaf water deficits upon photosynthesis, leaf conductance, water use efficiency, and osmotic potential in sorghum. Physiologia Plantarum 45: 103-111.

KHANNA \& R CHOPRA (1999) Osmotic adjustment and yield stability in wheat genotypes and species grown in water limited environments. Journal of Plant Biology 26: 173-178.

LUDLOW M, SANTAMARIA J \& FUKAI S (1990) Contribution of osmotic adjustment to grain yield in Sorghum bicolor under water-limited conditions. II Water stress after anthesis. Australian Journal of Agricultural Research 41: 67-78.

MORGAN JM (1983) Osmoregulation as a selection criterion for drought tolerance in wheat. Australian Journal Agricultural Research 34: 607-614.

MORGAN JM (1984) Osmoregulation and water stress in higher plants. Annual Review of Plant Physiology 35: 299-319.

MORGAN JM (1992) Osmotic components and properties associated with genotypic differences in osmoregulation in wheat. Australian Journal of Plant Physiology 19: 67-76.

MORGAN JM (1995) Growth and yield of wheat lines with different osmoregulative capacity at high soil water deficit in seasons of varying evaporative demand. Field Crops Research 40: 145-152.

REKIKA D, M NACHIT, J ARAUS \& P MONNEVEUX (1998) Effects of water deficits on photosynthetic rate and osmotic adjustment in tetraploid wheats. Photosyntetica 35: 129-138.

SANTIBÁÑEZ F \& J URIBE (1990) Atlas agroclimático de Chile. Ediciones de la Universidad de Chile, Santiago, Chile. 65 pp.

SEN GUPTA A \& G BERKOWITZ (1987) Osmotic adjustment, symplast volume, and nonstomatally mediated water stress inhibition of photosynthesis in wheat. Plant Physiology 85: 1040-1047.

TEULAT B, D REKIKA, M NACHIT \& P MONNEVUX (1997) Comparative osmotic adjustments in barley and tetraploids wheats. Plant Breeding 116: 6, 519-523.

TURNER NC \& M JONES (1980) Turgor maintenance by osmotic adjustment: a review and evaluation. En: Turner NC \& PJ Kramer (eds) Adaptation of plants to water and high temperature stress: 87-103. Wiley Inter Science, New York, New York, USA.

WILSON JR, MJ FISHER, ED SCHULZE, GR DOLBY \& MM LUDLOW (1979) Comparison between pressurevolume and dew point-hygrometry techniques for determining the water relations characteristics of grass and legume leaves. Oecologia 41: 77-88.

WRIGHT G, R SMITH \& J MORGAN (1983) Differences between two grain sorghum genotypes in adaptation to drought stress III. Australian Journal of Agricultural Research 34: 637-651.

ZADOKS JC, TT CHANG \& CF KONZAK (1974) A decimal code for the growth of cereals. Weed Research 14: 415-421. 